\title{
An Experimental Study on the Fuel Reduction Potential of Heavy Duty Vehicle Platooning
}

\author{
Assad Al Alam \\ Scania CV AB \\ 15187 Södertälje, Sweden \\ E-mail: assad.alam@scania.se
}

\author{
Ather Gattami \\ ACCESS Linnaeus Centre \\ Royal Institute of Technology \\ 10044 Stockholm, Sweden \\ Email: gattami@kth.se
}

\author{
Karl Henrik Johansson \\ ACCESS Linnaeus Centre \\ Royal Institute of Technology \\ 10044 Stockholm, Sweden \\ Email: kallej@ee.kth.se
}

\begin{abstract}
Vehicle platooning has become important for the vehicle industry. Yet conclusive results with respect to the fuel reduction possibilities of platooning remain unclear. The focus in this study is the fuel reduction that heavy duty vehicle platooning enables and the analysis with respect to the influence of a commercial adaptive cruise control on the fuel consumption. Experimental results show that by using preview information of the road ahead from the lead vehicle, the adaptive cruise controller can reduce the fuel consumption. A study is undertaken for various masses of the lead vehicle. The results show that the best choice with respect to a heavier or lighter lead vehicle depends on the desired time gap. A maximum fuel reduction of $4.7-7.7 \%$ depending on the time gap, at a set speed of $70 \mathrm{~km} / \mathrm{h}$, can be obtained with two identical trucks. If the lead vehicle is $10 \mathrm{t}$ lighter a corresponding 3.8-7.4\% fuel reduction can be obtained depending on the time gap. Similarly if the lead vehicle is $10 \mathrm{t}$ heavier a 4.3-6.9\% fuel reduction can be obtained. All results indicate that a maximum fuel reduction can be achieved at a short relative distance, due to both air drag reduction and suitable control.
\end{abstract}

\section{INTRODUCTION}

The traffic intensity is escalating in most parts of the world, making traffic congestion a growing issue. In parallel, the demand for transportation services is increasing. Vehicle platoons, also known as convoys, have become a vast research area to address these issues. By packing for instance, heavy duty vehicles (HDVs) close to each other, the total road capacity can be increased and emissions can be reduced.

Vehicle platooing, depicted in Fig. 1, has been widely recognized as a mean to reduce harmful exhaust emission from the engine. Presently, a lidar or radar based system is implemented by many vehicle manufacturers that enables vehicles to follow each other closely with the aid of an adaptive cruise control (ACC) system. Governing vehicle platoons by an automated control strategy, the overall traffic flow is expected to be improved [1]. Acceleration and braking is reduced, effectively reducing the vehicle emissions. Early theoretical work regarding vehicle platoons and corresponding control was presented in [2], [3] and [4], where the issue of string stability was raised. Further analysis was conducted

This paper was supported by Scania CV AB, VINNOVA - FFI, and the Swedish Research Council.

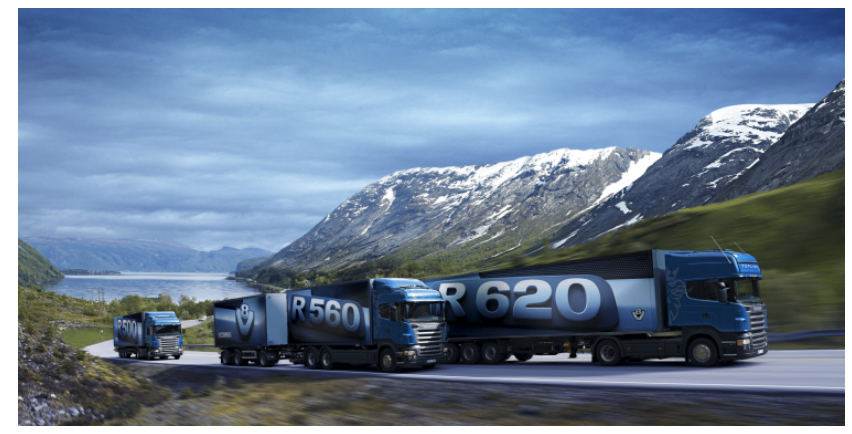

Fig. 1. Heavy duty vehicles traveling in a platoon can achieve significant fuel reduction.

in [5], [6], and [7]. The models representing the dynamics in these results were obtained by representing each vehicle as a simplified linear system with unity mass. For practical applications of vehicle platooning it is essential to use more realistic models. In HDV platooning mass and road slope has a significant effect on the vehicle dynamics. Thus, a more recent model with additional environment factors along with mass and road slope can be found in [8] and [9].

In this work, we are primarily concerned with the impact HDV platooning has on the fuel consumption. The focus within platooning so far has been on the environmental benefits and safety issues. It is of equal importance to investigate the economical prospective and feasibility of platooning for commercial purposes. Fuel cost constitutes approximately one third of the total operational cost in European long haulage HDVs [10]. Studies have shown that the wind resistance can be reduced significantly by arranging trucks in a platoon formation. It is partly due to that the drag produced behind the lead vehicle will be reduced when allowing a follower vehicle to lie close behind. The follower vehicle will experience a significant reduction of air drag due to a relatively large reduced pressure at the front. Hence, the total air drag is reduced, which in turn lowers the fuel consumption.

The main contribution of this paper is to investigate the fuel reduction potential of heavy duty vehicle platooning, 


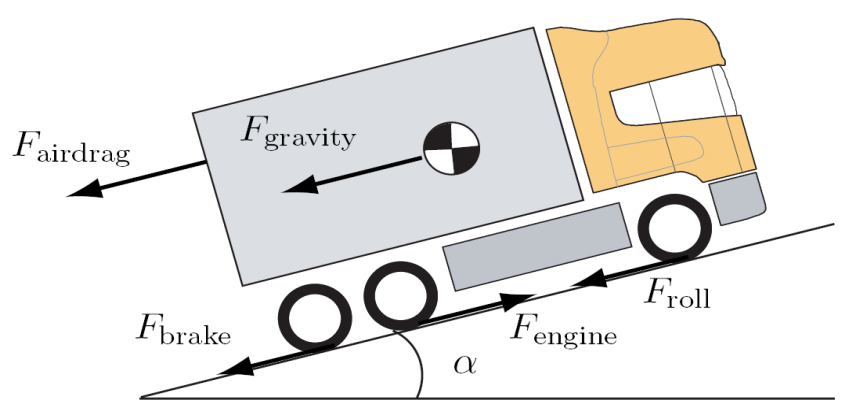

Fig. 2. The longitudinal forces inflicted upon a heavy vehicle in motion.

solely with respect to a commercial control strategy. The aim is not to investigate the specifics of the control strategy, which is left for future work. Fuel optimal control for a single vehicle on a flat road is to maintain a constant velocity, under the presumption that the traveling time is fixed [11]. Any deviations in the form of acceleration and deceleration result in an increased fuel consumption. An ACC generally receives information regarding the relative velocity and distance to the vehicle ahead and thereby maintains the relative distance by adjusting its speed accordingly. The increased control effort that the ACC creates, in the sense of additional transient engine actions and brake events, produces an overall increased fuel consumption. Thus to the best of our knowledge, it is still unclear whether the increased control effort produced by the ACC possibly cancels the reduction in fuel consumption achieved by decreasing the air drag.

The outline of the paper is as follows. First we give a description of the dynamics for heavy duty vehicles and platooning in Section II. In Section III we address the current technology in platooning applications and present a suitable system architecture for future applications. In Section IV empirical data obtained through field tests are utilized to validate the simulation model used in Section V. In Section V a method is derived to isolate the ACC's influence on the fuel consumption. Several case studies are presented to address the fuel reduction possibilities and to deduce the consequences of provoking the ACC behavior by having two vehicles of diffrent mass. Finally, in Section VI we present a brief summary of the results in this paper and conclusions.

\section{Problem Description}

We consider a platoon consisting of $N$ HDVs. Each vehicle has forces affecting it as depicted in Fig. 2. Its simplified equation of motion is given by:

$$
\begin{aligned}
m_{t} \frac{d v}{d t}= & F_{\text {engine }}-F_{\text {brake }}-F_{\text {airdrag }}(v) \\
& -F_{\text {roll }}(\alpha)-F_{\text {gravity }}(\alpha) \\
= & \frac{i_{t} i_{f} \eta_{t} \eta_{f}}{r_{w}} \mathcal{T}\left(\omega_{e}, \delta\right)-F_{\text {brake }}-\frac{1}{2} c_{D} A_{a} \rho_{a} v^{2} \\
& -c_{r} m g \cos \alpha-m g \sin \alpha
\end{aligned}
$$

where $\alpha$ denotes the slope of the road, $c_{D}$ and $c_{r}$ are characteristic coefficients, $g$ denotes the gravitational force,

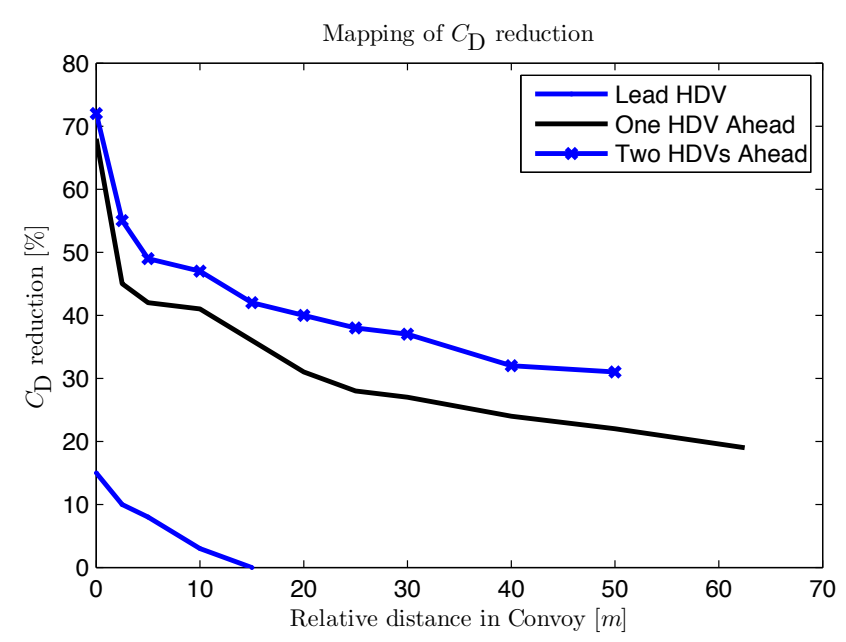

Fig. 3. Change in air drag coefficient $c_{D}$ and distance between the vehicles. Adapted from [12]. Similar findings have been confirmed by the fluid dynamics department at Scania CV AB and in [13].

$\rho_{a}$ the air density, $r_{w}$ the wheel radius, and $i_{t}, i_{f}, \eta_{t}, \eta_{f}$ are transmission and gear specific constants. The accelerated mass of the truck $m_{t}\left(m, J_{w}, J_{e}, i_{t}, i_{f}, \eta_{t}, \eta_{f}\right)$ depends on the gross mass $m$, wheel and the engine inertia $J_{w}, J_{e}$ and the constants above.

The aim of this study is to determine the fuel reduction possibilities for a platoon of $N$ HDVs traveling on a road with a given set speed and relative distance. We want to separate the fuel reduction obtained through the reduction in air drag and the fuel consumption produced by the commercial ACC effort. The HDVs are controlled based on the relative distance and velocity between the two vehicles. The reduction in the air drag coefficient is modeled by a graphical model presented in Fig. 3.

It is difficult to make an accurate deduction merely based on empirical results due to the varying external disturbances. E.g. weather conditions might vary and traffic conditions might change-producing incomparable and inconclusive empirical results with respect to the fuel consumption.

Note that the behavior of a HDV differs significantly from a light vehicle. Physical constraints have a larger impact on the vehicle dynamics due to the higher mass and inertia.

\section{Control System Architecture}

To achieve fuel reduction through platooning, the vehicles must drive at a close relative distance. This can only be obtained through tight control using sensor and wireless communication. In this section we present a brief description of the ACC and a new control system architecture, which has the potential to achieve the requirements for platooning.

\section{A. Adaptive Cruise Control}

The ACC generally acts as an extension to the CC. If a target is not detected or the leading vehicle is traveling faster than the set speed of the $\mathrm{CC}$, the ACC will not engage. If a target is located within the same lane and traveling with the same speed or slower, the ACC will adapt the relative 


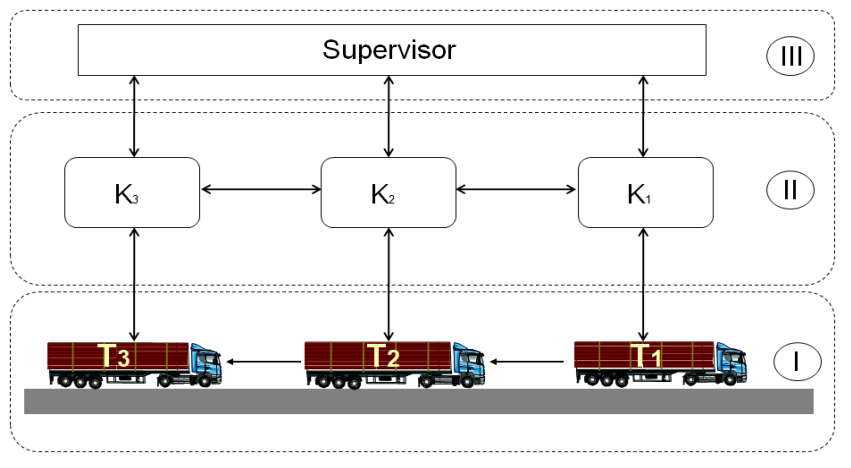

Fig. 4. System Architecture: The figure depicts three layers with arrows indicating the direction of information flow. The wireless communication node for each vehicle is denoted $K_{i}, \mathrm{i}=1,2,3$.

distance according to the desired time gap setting. It will not adjust the speed with respect to vehicles in separate lanes. When the leading vehicle switches lanes (turns off), the ACC will search for a new target accordingly. If a target is not detected, the system returns to $\mathrm{CC}$ constant speed mode.

Furthermore, a commercial ACC is mainly designed to maintain the desired relative distance in a comfortable manner by sending appropriate requests to the engine and the various brake systems present in a truck. It is not designed with respect to fuel optimal criteria. The relative distance is determined by letting the driver set a desired time gap $1, \ldots, 5$, which is proportional to the relative distance, where time gap 1 corresponds to the shortest relative distance. A short relative distance will require the ACC to perform more stringent control actions due to safety issues.

\section{B. Architecture}

The need for a proper architecture has been studied in [14]. We propose a new three-layer hierarchical control system architecture, depicted in Fig. 4. It is mainly designed with respect to control applications within platooning.

Starting from the bottom, layer I consists of feedback information regarding the relative distance and velocity of the vehicle ahead obtained through a radar sensor. The task within this layer is mainly safety through collision avoidance with the vehicle ahead as well as fuel efficient control decisions.

Layer II, expands the decision space of the controller with the introduction of wireless nodes. Its task is to provide the ACC with information regarding the intents and behavior of the surrounding vehicles. Thus the ACC will able to make decentralized fuel optimal decisions based on vehicles within spatial range of its wireless tranciever, without compromising safety.

Lastly, layer III extends the decision space by adding a third dimension into the decision space. Here dynamic route planning, route assigning, platoon formation, route topology, etc. will be provided by wireless information to the ACC. Hence the ACC will be able to make strategic decisions based on future and global events, further improving the fuel efficiency.
TABLE I

FUEL REDUCTION FOR A TWO VEHICLE PLATOON.

\begin{tabular}{ccc} 
& $\begin{array}{c}\text { Simul. Fuel } \\
\text { consump. [\%] }\end{array}$ & $\begin{array}{c}\text { Exper. Fuel } \\
\text { consump. [\%] }\end{array}$ \\
\hline Lead Truck & 100 & 100 \\
Time Gap 1 & 93.2 & 92.9 \\
Time Gap 3 & 94.9 & - \\
Time Gap 5 & 98.8 & 98.7 \\
\hline
\end{tabular}

The technology needed to implement the three layers are already available. However, certain issues must be evaluated before commercialization is feasible. Therefore, in this paper, we have chosen to focus on the premises mentioned for layer I, i.e. the radar based ACC, to address whether a contemporary commercial ACC is fuel efficient in platooning applications.

\section{EXPERIMENTS}

The main difficulty lies in producing an environment where reproducible results can be obtained. In this section we present a method to obtain data empirically for $N=2$ HDVs.

\section{A. Setup}

Experiments were conducted upon a Swedish highway with two identical trucks as illustrated in Fig. 1. The masses of the heavy duty vehicles was measured to be $39.3 \mathrm{t}$ and $39.2 \mathrm{t}$. Both of them were equipped with a Scania $620 \mathrm{hp}$ engine. The fuel consumption was measured and recorded through each vehicles CAN-devices. It can be measured for two trucks traveling on the road, but to facilitate a correct deduction, the ambient variables, e.g., wind, ambient temperature, humidity, etc., must be equal during every measurement instance (test-run) to create reproducible results. Small variations in these disturbances can produce a significant difference in measured fuel consumption. An additional reference truck was therefore used as a calibration device to reduce the error due to the varying environment factors. All the vehicles were traveling with a set speed of $90 \mathrm{~km} / \mathrm{h}$. Several runs were conducted for which the results are presented in Section IV-B.

\section{B. Results}

The empirical data obtained from field tests was used to validate the accuracy and validity of the simulation model used in Section V. The results are presented in Table I, where fuel consumption data is provided for test-runs with varying time gap settings $1,3,5$. Simulated fuel consumption is given together with experimental fuel consumption. The lead truck was governed by a CC and used as a reference.

The results in Table I verify that a significant fuel reduction can be obtained through platooning and the simulated values correspond very well to the empirical results. The findings presented in Fig. 3 were however derived from experiments conducted on buses in a wind tunnel. Hence no effects from additional aerodynamics, e.g., lateral winds, were taken into consideration. Convoy driving does not reduce the resistance from such winds. Thus, only $80 \%$ of 
the suggested values was utilized and assumed to give a good estimate of reasonable air drag reduction within platooning applications. The slightly lower values in fuel reduction are most likely a result of the aforementioned assumption. Therefore it can be deduced that the results produced by the simulation are most certainly reliable and mirrors real truck behavior quite accurately.

\section{Simulation}

In this section we first present a model and then a method to separate the fuel reduction obtained by the reduction in air drag and the fuel consumption produced by the commercial ACC effort. Lastly, we investigate the impact on fuel reduction by varying the mass of the HDVs.

\section{A. Model}

To create a simulation environment, which produces reliable results and mirrors real-life behavior, an advanced model for two coupled HDVs is developed as an extension to the acausal nonlinear model for a single vehicle created by [15].

Each individual part in the powertrain, such as engine, gearbox, clutch, etc., is modeled in detail, simulated, and verified to mimic real-life behavior, resulting in a model consisting of 3313 variables, 1058 equations, and 626 states. It was constructed in a modeling tool called Dymola [16]. Dymola enables a bidirectional data flow, which in turn makes it possible to model and simulate acceleration as well as coasting. In the process of acceleration, the force produced by the engine flows towards the road surface, whereas it is reversed during coasting.

The advanced truck model for a single vehicle is duplicated and modified to model a vehicle with $\mathrm{CC}$ and another vehicle with ACC as depicted in Fig. 6. ACC- and CC-logic to govern the trucks was implemented by utilizing the current software implemented in Scania CV AB's trucks in production. The advanced truck model with the CC/ACC-software was also tested and verified to represent characteristic and reliable behavior observed in real trucks.

Results in this study were obtained by empirical results and by simulating a truck with a $620 \mathrm{hp}$ engine and a 12 speed gear box. The vehicle configuration was $6 \times 2$ and the gross mass of the truck was chosen to be $40000 \mathrm{~kg}$, i.e the estimated standard average weight of the European long haulage trucks.

To obtain results based upon conditions that represent reallife scenarios, a fairly hilly road was selected as a simulation basis. The Swedish road between Södertälje and Jönköping, depicted in Fig. 5, is considered to characterize a varied range of road conditions.

\section{B. Results}

Trucks have a speed restriction by law upon many roads; hence $70 \mathrm{~km} / \mathrm{h}$ was considered to be a reasonable set speed for the lead vehicle's CC, as depicted in Fig. 6.

The set speed for the follower truck with ACC was set at $80 \mathrm{~km} / \mathrm{h}$ to inhibit a possible loss of the leading vehicle due to factors in the topology. Hence, the results from simulating

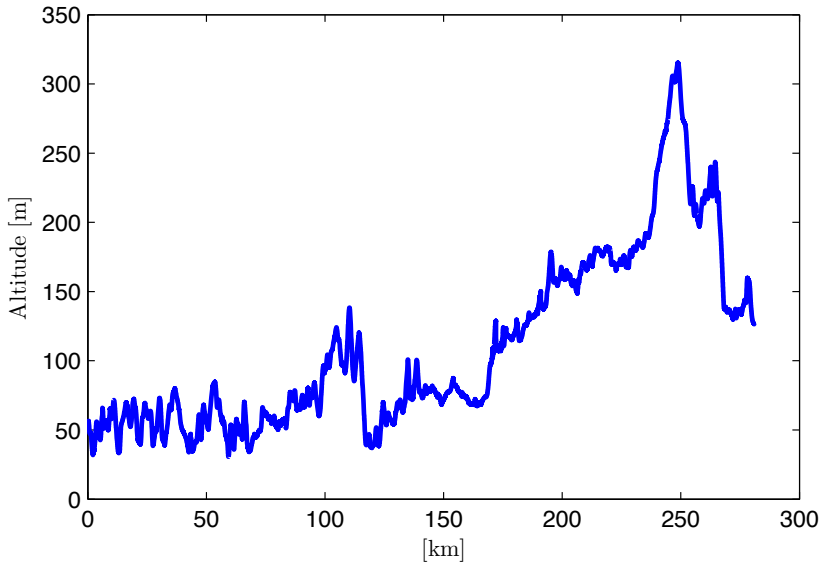

Fig. 5. Topology for the road Södertälje - Jönköping, Sweden

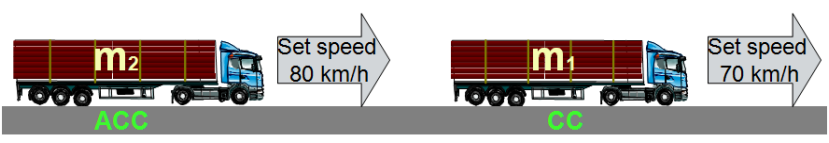

Fig. 6. Simulation scenario.

two identical trucks on the afore mentioned road are stated in Table II for various time gaps.

The results clearly show that a reduction of $4.7-7.7 \%$ in fuel consumption is attainable with a ACC compared to a truck with a conventional CC. It should also be noted that the fuel reduction is obtained without reducing the average velocity.

\section{Isolating the Influence from the ACC}

The simulation in Section V-B shows an overall fuel reduction can be obtained when driving two trucks in a platoon. Hence, it has become evident that the general control effort produced by the ACC to maintain the relative distance between the vehicles does not cancel the total fuel reduction possibilities obtained through the reduced air drag. However, it is unclear whether the ACC effort produces an increase or decrease of fuel consumption.

To separate and determine the effect of the control effort on the fuel consumption, all other factors must be kept constant. Hence, the truck parameters, i.e. engine, gear box, weight, rolling resistance, etc., are set equal for both vehicles-eliminating any possibilities for the fuel consumption to differ due to different physical properties between the trucks. Furthermore, to facilitate a correct deduction, the ambient variables, i.e. ambient temperature, humidity, traffic conditions, etc., and the air drag, are also set equal for both vehicles, effectively isolating the effect of the control strategy on the fuel consumption within the simulation model depicted in Fig. 6.

The simulation results for different time gaps presented in Table III, show that the overall control effort actually reduces the fuel consumption. Maximum reduction in fuel 
TABLE II

NORMALISED RESULTS FROM SIMULATION WITH IDENTICAL TRUCKS

\begin{tabular}{ccc} 
& $\begin{array}{c}\text { Fuel } \\
\text { consumption [\%] }\end{array}$ & $\begin{array}{c}\text { Average } \\
\text { Velocity }[\mathrm{km} / \mathrm{h}]\end{array}$ \\
\hline Lead Truck & 100 & 69.89 \\
Time Gap 1 & 92.3 & 69.90 \\
Time Gap 3 & 93.6 & 69.90 \\
Time Gap 5 & 95.3 & 69.89 \\
\hline
\end{tabular}

TABLE III

NORMALISED RESULTS FROM SIMULATION WITH IDENTICAL AIR DRAG

\begin{tabular}{ccc} 
& $\begin{array}{c}\text { AND TRUCKS } \\
\text { Fuel } \\
\text { consumption [\%] }\end{array}$ & $\begin{array}{c}\text { Average } \\
\text { Velocity [km/h] }\end{array}$ \\
\hline Lead Truck & 100 & 69.89 \\
Time Gap 1 & 98.8 & 69.90 \\
Time Gap 3 & 98.9 & 69.89 \\
Time Gap 5 & 99.1 & 69.89 \\
\hline
\end{tabular}

consumption solely with respect to the control strategy is obtained on time gap 1 . There is a slight but insignificant change in fuel consumption for time gap 5.

A possible explanation for this discovery is derived by studying a segment of the road. Fig. 7 shows the vehicle behavior for both of the trucks controlled with CC and ACC. The top plot shows the actual vehicle velocity trajectory of the two trucks, with implied speed requests. When traveling along a down hill, the ACC-logic allows the subject vehicle to increase its speed and thereby decrease the relative distance. When exiting the downhill the ACC deters the preservation of the set speed, thus effectively increasing the relative distance. It becomes evident by studying the instantaneous fuel consumption (Fig. 7: second plot from the top) at the $5780 \mathrm{~m}$ marker and the $6040 \mathrm{~m}$ marker that such a control strategy reduces the fuel consumption. The area between the curves at the aforementioned markers represent the saved fuel. Studies in economical cruise control strategy [17], have also proven that utilizing the gravitational force obtained in a downhill to increase the velocity and utilizing the provided energy to deter control actions until mandated, reduces fuel consumption. Therefore the observed behavior can be characterized as enabling classical economical cruise control behavior through preview information of the road characteristics ahead from the lead vehicle, which undoubtedly reduces the fuel consumption.

The noted result could also have been a consequence of finding a more fuel-efficient Fuel-Torque-RPM point for the specific engine due to a small difference in actual speed between the two trucks. However, an identical simulation with a different $620 \mathrm{hp}$ engine resulted in equivalent results concluded from Table III.

\section{Different Mass}

Owing to the aforementioned results, they indicate that platooning with two identical trucks reduces the fuel consumption due to air drag reduction and control strategy. However, two trucks with different mass could induce a different ACC behavior. In some cases when following a
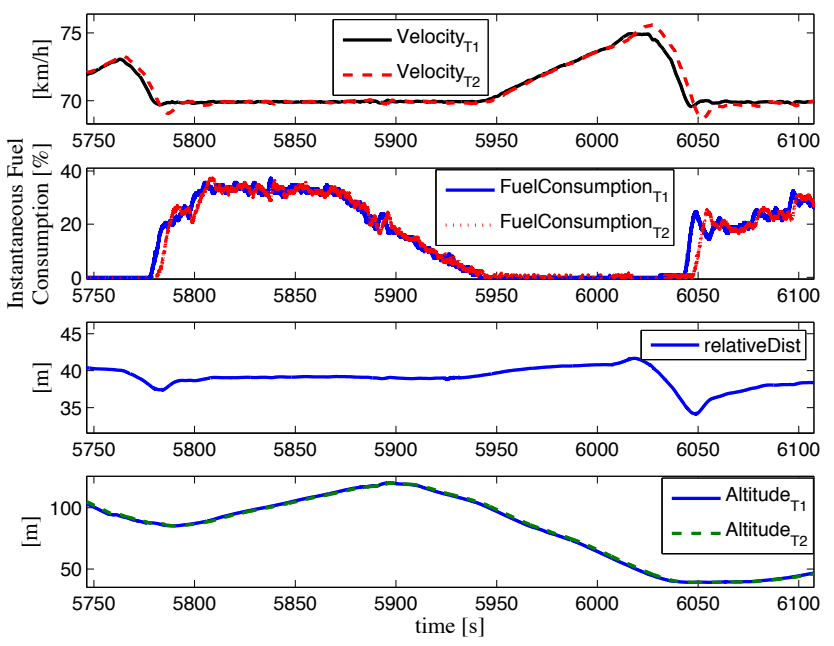

Fig. 7. Simulation results: Top plot: Velocity trajectory for the first truck controlled by CC, sub indexed T1, (solid line) and the second truck governed by ACC, sub indexed T2 (dashed line). Second plot from the top: Instantaneous fuel consumption for T1 (solid line) and T2 (dashed line). Third plot from the top: Relative distance between the vehicles (solid line). Bottom plot: Topology for T1 (solid line) and T2 (dashed line).

lighter truck in an uphill, the lead vehicle will be able to maintain its velocity, while the follower vehicle will decline in speed due to its extensive mass. Similarly when following a heavier lead vehicle, the follower vehicle will have to accelerate while traveling along a steep downhill to maintain the relative distance. Hence, a difference in mass between the two trucks will inflict constraints on the ACC, i.e. altering the controller behavior in comparison with the case of two identical trucks. Thus it is of interest to study the effects of provoking the ACC strategy in such a manner.

Two scenarios were investigated. In the first scenario, the mass of the lead vehicle is set to $m_{1}=30 \mathrm{t}$, whereas the truck governed by ACC is maintained at $m_{2}=40 \mathrm{t}$, see Fig. 6 . In the second scenario, the leading vehicle's mass is set to $m_{1}=$ $50 \mathrm{t}$. All other conditions are identical and the simulation is carried out on the same road as before.

The results displayed in Fig. 8 were derived by comparing the fuel consumption of the follower vehicle, with the fuel consumption of a lead vehicle with the same mass to avoid ambiguity. Fig. 8 reveals a fuel reduction of $3.8-7.4 \%$, while following a lighter lead vehicle and a reduction in fuel consumption of $4.3-6.9 \%$, when following a heavier lead vehicle. The results show that a significant fuel reduction can still be obtained with various time gaps. However a noticeable difference in fuel consumption is detected due to the physically induced change in control. For time gap 1, a $0.5 \%$ higher fuel reduction can be obtained with a lighter lead vehicle in comparison with a heavier lead vehicle. It is probably due to the fact that the vehicle governed by ACC acts as a low pass filter when it is heavier, making it insensitive to small fluctuations in the lead vehicle's velocity. Thus less variance in control effort is implemented on the vehicle, resulting in lower fuel consumption. However, on 


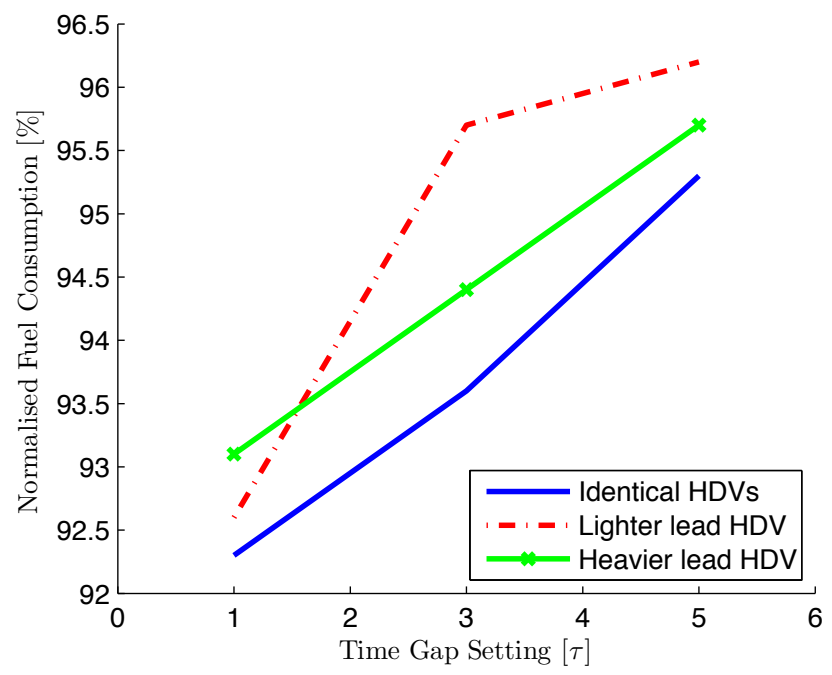

Fig. 8. The figure illustrates the results of simulating platooing with two trucks of different mass and identical mass.

time gap 5 a lighter lead vehicle produces an increase of $0.5 \%$ in fuel consumption compared to the results from a heavier lead vehicle. A lighter lead vehicle will be able to maintain its speed with less effort in an uphill. Thus, the heavier truck governed by the ACC will need more effort to maintain the relative distance. Hence, more fuel is injected to the system to reduce the relative distance. When the lead vehicle is heavier, fewer situations will arise when the relative distance needs to be reduced. If such situations arise, less effort will be needed.

\section{CONCLUSION}

In this paper we have shown that a maximum fuel reduction of $4.7-7.7 \%$ depending on the time gap, at set speed equal to $70 \mathrm{~km} / \mathrm{h}$, can be obtained with two identical trucks. If the lead vehicle is $10 \mathrm{t}$ lighter a corresponding 3.8-7.4\% fuel reduction can be obtained depending on the time gap setting. Similarly if the lead vehicle is $10 \mathrm{t}$ heavier a $4.3-$ $6.9 \%$ fuel reduction can be obtained. The fuel consumption in a N $>2$ HDV platoon can most likely be lowered further due to an additional reduction in air drag for additional vehicles. All results indicate that a maximum fuel reduction will be achieved with time gap 1, due to both reduction in air drag and the control strategy.

The ACC-strategy does not increase the fuel consumption according to the results obtained in this study. By naturally mirroring economical cruise control behavior through preview information, the ACC becomes a fuel efficient control strategy, which produces approximately $1 \%$ fuel reduction solely based upon the ACC actions. Surprisingly, the more stringent control effort implemented on time gap 1 does not increase the overall fuel consumption. The results clearly show that no significant difference in fuel reduction occurs between time gaps with respect to control effort. However, a vast reduction of air drag can be obtained by reducing the time gap, i.e. reducing the relative distance. Hence, it is favorable to minimize the relative distance between the vehicles to achieve a maximum reduction in air drag. However issues such as safety and driver comfort arises. Thus a need for further investigation within the subject matter arises.

The analysis conducted in this study shows that improvements can be made to the ACC-logic by designing it based upon fuel optimal criteria. Owing to the fact that the ACC-strategy produces different fuel reduction results when following a truck of different mass and to the fact that isolated strategies within this paper have shown fuel reduction possibilities, there is a vast scope of designing a fuel optimal controller for commercial purposes without compromising comfort and safety issues.

\section{ACKNOWLEDGMENTS}

The authors would like to thank J. Andersson, H. Flemmer, T. Nyström, Å. Nylander, T. Sandberg, and F. Stensson at Scania CV AB, for their support and contribution.

\section{REFERENCES}

[1] P. A. Ioannou and C. C. Chien, "Autonomous intelligent cruise control," IEEE Transactions on Vehicular Technology, vol. 42, NO. 4, November 1993.

[2] W. Levine and M. Athans, "On the optimal error regulation of a string of moving vehicles," IEEE Transactions on Autmatic Control, vol. AC11, no.3, pp. 355-361, 1966.

[3] S. M. Melzer and B. C. Kuo, "Optimal regulation of systems described by a countably infinite number of objects," Automatica, vol. 7, pp. 359-366, 1971.

[4] J. L. Willems, "Optimal control of uniform string of moving vehicles," Ricerche di automatica, vol. 2, pp. 184-192, 1971.

[5] D. Swaroop and J. Hedrick, "String stability of interconnected systems," IEEE Transactions on Automatic Control, 1996.

[6] M. R. Jovanović and B. Bamieh, "On the ill-posedness of certain vehicular platoon control problems," Proceedings of the 43rd IEEE Conference on Decision and Control, 2004.

[7] J. Rogge and D. Aeyels, "Decentralized control of vehicle platoons with interconnection possessing ring topology," 44th IEEE Conference on Decision and Control, and the European Control Conference, pp. 1491-1496, 2005.

[8] P. Sahlholm and K. H. Johansson, "Road grade estimation for look-ahead vehicle control using multiple measurement runs," Control Engineering Practice, vol. In Press, Corrected Proof, pp. -, 2009. [Online]. Available: http://srxxw.com/science/article/B6V2H4XK31R9-1/2/ac2fe73048eb40aa867b1f9e5bafd706

[9] L. Guzzella and A. Sciarretta, Vehicle Propulsion Systems, 2nd ed. Springer Berlin Heidelberg New York, 2007, ISBN: 978-3-540-746911.

[10] Scania CV AB, Annual Report, Scania CV AB, 2001.

[11] E. Hellström, J. Åslund, and L. Nielsen, "Design of a well-behaved algorithm for on-board look-ahead control," in IFAC World Congress, Seoul, Korea, 2008.

[12] H. Wolf-Heinrich and S. R. Ahmed, Aerodynamics of Road Vehicles. Warrendale: Society of Automotive Engineers, Inc, 1998.

[13] C. Bonnet and H. Fritz, "Fuel consumption reduction in a platoon: Experimental results with two electronically coupled trucks at close spacing," SAE paper 2000 - 01 - 3056, 2000.

[14] P. Varaiya, "Smart cars on smart roads: Problem of control," IEEE Transactions on Automatic Control, vol. 38, NO. 2, February 1993.

[15] T. Sandberg, Heavy Truck Modeling for Fuel Consumption, Simulations, and Measurements. S-581 83 Linköping, Sweden: Licentiate thesis LiU-TEK-LIC-2001:61, Linköpings universitet, 2001.

[16] Dynasim, User Manual Dymola 6 Additions, Dynasim AB, Research Park Ideon, SE-223 70 Lund, Sweden, June 2007.

[17] E. Hellström, A. Fröberg, and L. Nielsen, "A real-time fuel-optimal cruise controller for heavy trucks using road topography information," SAE paper 2006-01-0008, 2006. 